\title{
Clavien-Dindo Classification
}

National Cancer Institute

\section{Source}

National Cancer Institute. Clavien-Dindo Classification. NCI Thesaurus. Code C121446.

A classification of adverse events that grades complications according to the treatment that is induced to treat the complication. The classification is designed to eliminate subjective interpretation of serious adverse events and tendency to down-rate complications, being based on data that is usually well documented and easy to verify. It is also well suited for retrospective analyses. 